\title{
MAPA - Monitorização ambulatorial da pressão arterial
}

\author{
F. NOBRE, D. MION J R.
}

Divisão de Cardiologia do Hospital das Clínicas da Faculdade de Medicina de Ribeirão Preto da Universidade de São Paulo, Ribeirão Preto, SP; Disciplina de Nefrologia do Hospital das Clínicas da Universidade de São Paulo, São Paulo, SP.

UNITERMOS: Monitorização ambulatorial da pressão arterial. Hipertensão.

KEY WORDS: Arterial blood pressure monitoring. Hypertension.

Monitorização ambulatorial da pressão arterial (MAPA) é uma técnica que permite obter medidas múltiplas e indiretas da pressão arterial durante 24 ou mais horas consecutivas com um mínimo de desconforto, durante as atividades diárias do paciente ${ }^{1}$.

A possibilidade de se obter uma curva representativa das variações pressóricas em determinado período oferece uma visão dinâmica do comportamento tensi onal e não apenas uma observação meramente estática, refletindo somente o instante em que foi medida a pressão arterial ${ }^{2}$.

A utilização da MAPA permite estudar o padrão normal de pressão arterial| ${ }^{3,4}$, as lesões em órgãosalvo decorrentes da hipertensão ${ }^{5}$, o prognóstico de eventos cardiovasculares e mortalidade ${ }^{6,7}$ e a análise da eficácia das drogas anti-hipertensivas ${ }^{8-10}$.

A MAPA é, hoje, um procedimento efetivamente incorporado à prática clínica.

Em 1987, Garret e Kaplan ${ }^{11}$, em editorial do J ournal of Clinic Hypertension, posicionaram a MAPA como um procedimento promissor e chei o de ótimas perspectivas nas suas aplicações. Recentemente, o board of trustees do American College of Cardiology aprovou parecer de uma comissão de renomados especialistas em hipertensão, sob o título "ACC Position Statement - Ambulatory Blood Pressure Monitoring"12, que, em resumo, conclui: "A MAPA tornou-se um método maduro, clinicamente apli cável, com normatizações desenvolvidas por importantes sociedades e com consensos americanos e internacionais para suas indicações e procedimentos".

Por essas características do método e pelo momento pelo qual passa, justifica-se a abordagem desse importante assunto.

As principais vantagens e as limitações da monitorização ambulatorial em relação às medidas casuais da pressão arterial são mostradas na tabela 1.

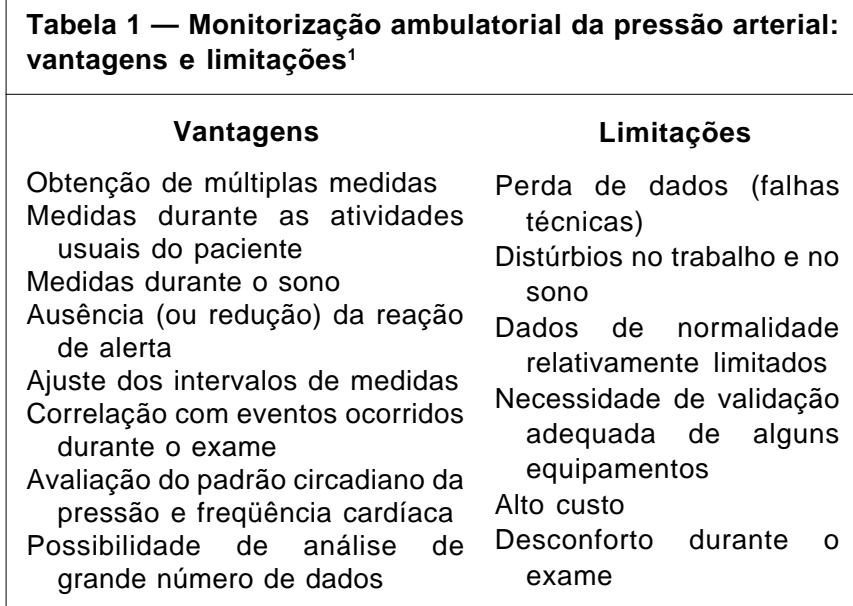

Tabela 2 - Principais indicações da monitorização ambulatorial da PA $^{1}$

Hipertensão "limítrofe" com lesão de órgãos-alvo

Hipertensão do avental branco (hipertensão de consultório)

Avaliação da hipertensão resistente ao tratamento

Hipertensão episódica (e.g. feocromocitoma)

Síncopes

Hipotensão sintomática associada a medicamentos anti-hipertensivos

Disfunção autonômica

Avaliação do comportamento pressórico na angina noturna e na congestão pulmonar

Avaliação de eficácia terapêutica

As situações clínicas em que a MAPA tem utilidade e, portanto, indicação estão expressas na tabela 2 .

\section{UTILIZAÇÃO DA MAPA NA PRÁTICA CLÍNICA}

Hipertensão "limitrofe" com lesão de órgãos-alvo

Ocasionalmente, indivíduos que apresentam pressão do tipo "normal alto" (valores entre 85 e $89 \mathrm{mmHg}$ ) e que têm lesões em órgãos-alvo (e.g. hipertrofia ventricular esquerda, retinopatia etc.) podem, quando submetidos à MAPA, mostrar níveis de pressão significativamente mais el evados relaci onados a estresse, esforço físico e/ou mental, tipo de trabalho, evidenciando, nesses casos, que a pressão obtida no consultório foi subestimada. 


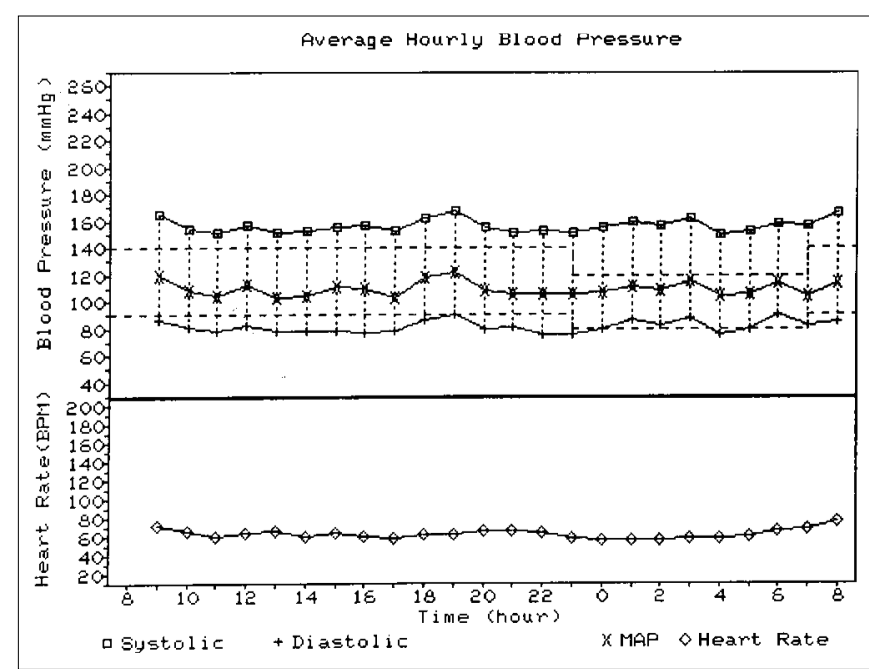

Fig. 1 - Monitorização ambulatorial da pressão arte rial de 24 horas mostrando el evação sistemática da pressão arterial sistól ica. Observa-se ausência de queda da pressão entre os períodos de vigília e sono. Este fato, quando presente em paci entes hi pertensos, pode rel acionar-se com mai or probabili dade de compli cações cardi ovasculares e lesão em órgãos-alvo, constituindo-se em importante marcador prognóstico.

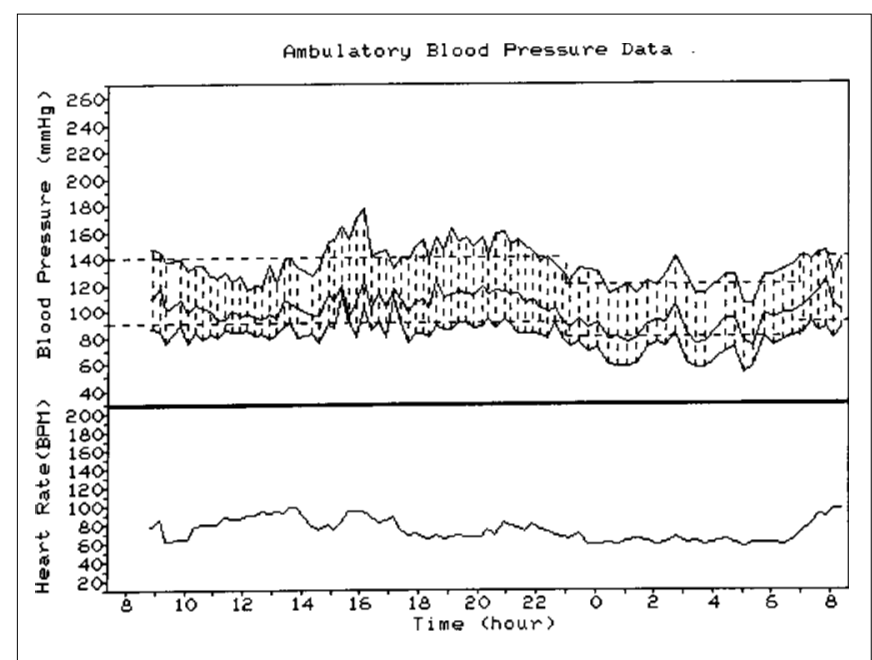

Fig. 2 - MAPA em paciente portadora de feocromocitoma produtor denoradrenal ina. Observa-sepresença depi cos tensi onai s eal ta variabili dade da pressão. A anál isedas médias tensionais entre os períodos de vigília e sono revel ou descenso atenuado, comum nos casos de hipertensão secundária.

Hipertensão do avental branco ou hipertensão do consultório

Pickering e O'Brien ${ }^{13}$ afirmam, categoricamente, que a mais importante das apli cações clínicas da MAPA consiste na possibilidade de avaliar, adequadamente, os pacientes que apresentam um comportamento anormal da pressão quando examinados no consultório, mas não o reproduzem na observa-

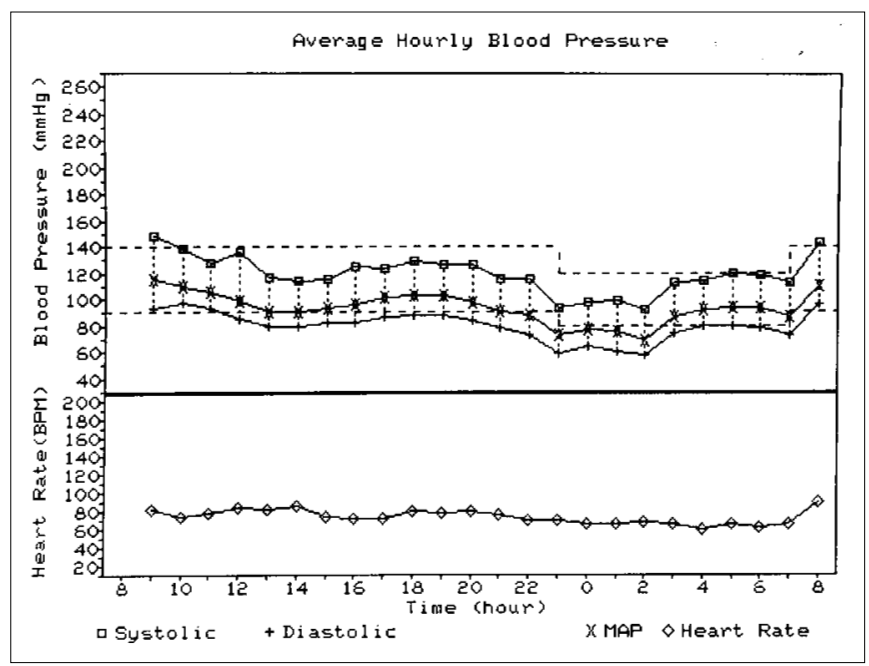

Fig. 3 - Paciente apresentando níveis tensionais sistematicamente el evados em medidas casuais. Revelava, entretanto, medidas domiciliares normais. MAPA demonstrando el evação da pressão somente no início e término do exame (medidas que habi tual mente são realizadas na clínica), com comportamento normal da pressão nas 24 horas. Trata-sedeum casotípi co de "hi pertensão do avental branco".

ção de 24 horas. Krakoff et al. ${ }^{14}$ estimam que o número de pacientes com hipertensão leve, em tratamento medicamentoso anti-hipertensivo, poderia ser reduzido em torno de $25 \%$, sem que a mortalidade aumentasse.

Não há dúvidas de que essa possibilidade atraente e prática de se excluir um contingente aproximado de $20 \%$ a $30 \%$ dos hipertensos leves e moderados qualifica a MAPA como método dos mais úteis para esse fim.

Alguns estudos suger em ${ }^{15,16}$ que parte desses pacientes que têm pressão arterial al terada no consultório com curvas de pressão de 24 horas normais não precisam ser tratados porque não reproduzem, em suas atividades habituais, o mesmo comportamento tensional observado diante do médico, devendo, entretanto, ser seguidos clinicamente.

Recentemente, Pierdomenico et al. ${ }^{17}$ demonstraram que esse grupo de indivíduos, em função de uma evolução rel ativamente benigna, sem comprometimento de orgãos-alvo, poderá ter o seu tratamento medicamentoso postergado para o momento, se este ocorrer, em que houver evidências de uma hipertensão estabelecida.

Durante o Congresso Mundial de Hipertensão, realizado em 1994, em M el bourne, Austrália, vários trabalhos sugeriam que esses pacientes podem apresentar mai or probabilidade de compli icações decorrentes de hipertensão arterial, como alterações de órgãos-alvo e microal buminúria, por exemplo.

Esses aspectos, ainda por resolver, deverão ser 


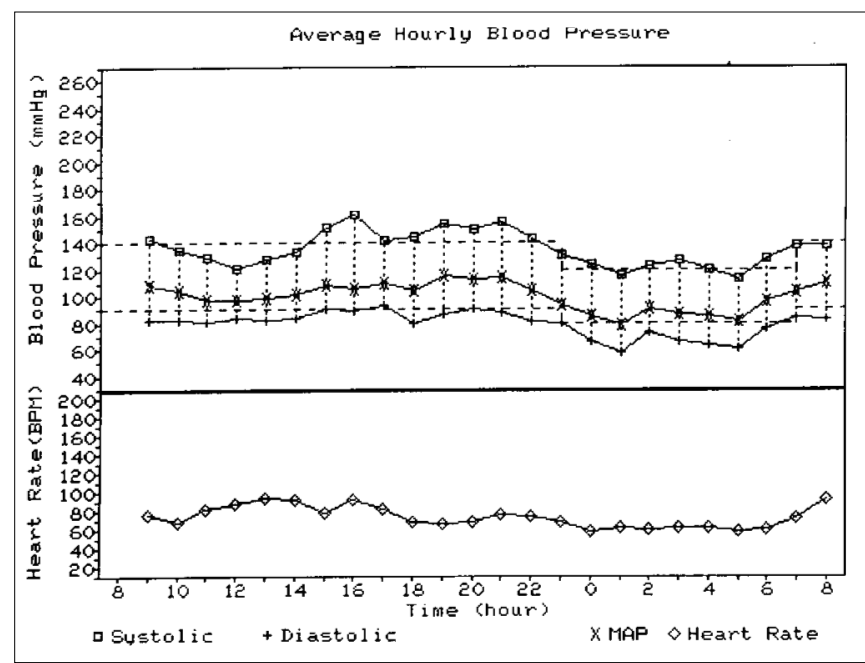

Fig. 4 - Exame real izado para aval iação da efi cácia da terapêutica em uso. Podese observar que a paciente mantém pressão dentro dos l imites normais até por vol ta de 15:00 horas, quando há escape do controle tensi onal, permanecendo a pressão si stól i ca el evada até 22:00 horas. Nessas circunstâncias, a monitorização pôde indicar a necessidade de ajuste terapêutico para controle adequado e contínuo da pressão nas 24 horas.

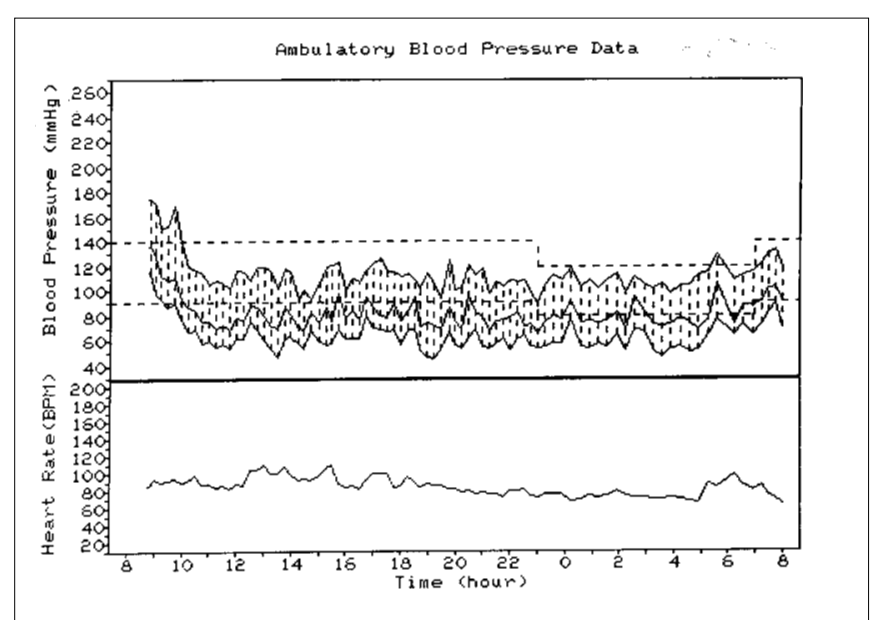

Fig. 5 - Neste caso, a situação clínica é semel hante à relatada na fig. 3. Observa-se, entretanto, a acentuada resposta hipertensiva no início do exame, em contraposições a um comportamento tensi onal dentro dos limites da normalidade durante o restante da MAPA.

motivo de estudos prospectivos de grande escala para que tenhamos as respostas necessárias e esperadas.

\section{Hipertensão resistente à terapêutica}

Considera-se que a hipertensão é resistente ao tratamento quando não há resposta adequada pelas avaliações de consultório, em um paciente submetido a tríplice terapia. Entretanto, muitas vezes, o efeito "avental branco" é o responsável por

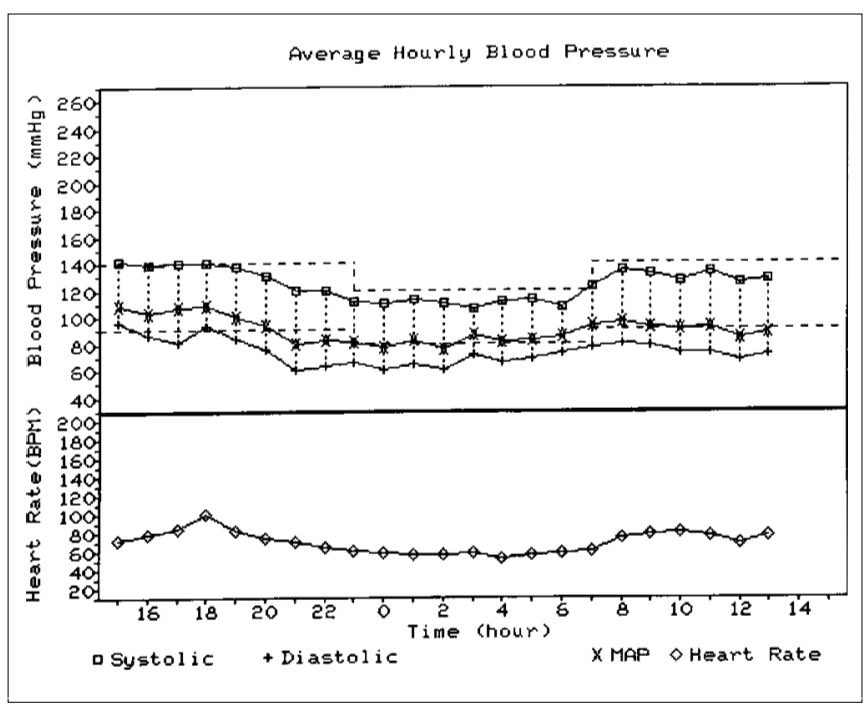

Fig. 6 - Paciente apresentando, durante as consultas médicas, níveis tensi onais el evados a despei to do uso regular da medicação prescrita. A monitorização da pressão em 24 horas mostrou que a pressão se mantinha dentro dos limites normais. Trata-se de um caso em que se observa o chamado "efeito do avental branco".

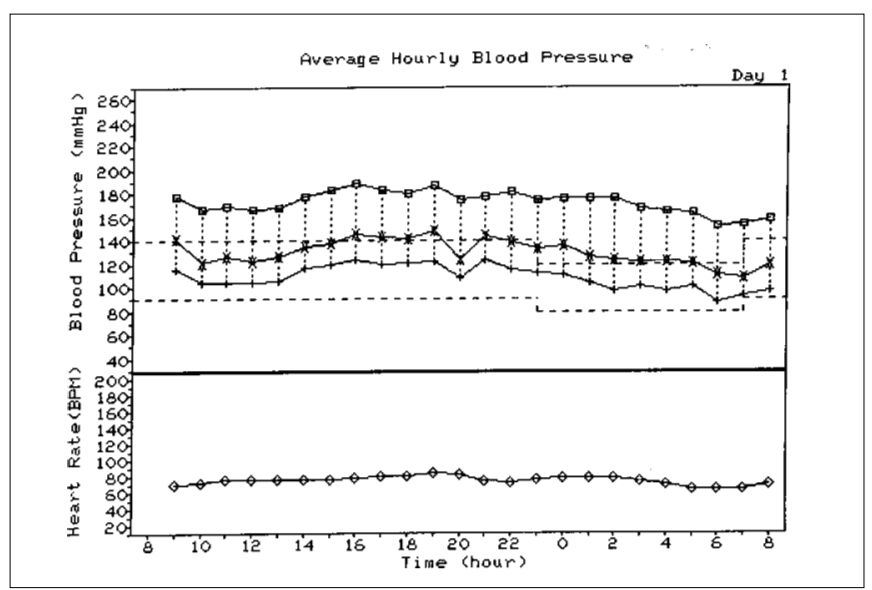

Fig. 7 - Examerealizado para aval iação de efi cácia da terapêuti ca em uso. Observa-se claramente que o esquema posológi co vigente não está sendo adequado para a manutenção da pressão nos níveis desejados. Pode-se, também, observar que não há descenso fi siológico esperado da pressão entre a vigília e o sono.

esta inadequada resposta hipotensora observada no consul tório. Se há indícios de que a pressão está controlada, por exemplo, mediante avaliações domiciliares, a MAPA está indicada para a análise da eficácia da medicação em uso. Há, também, que se considerar as características individuais de cada paciente diante da ação dos medicamentos que estão sendo utilizados, fato no qual também a monitorização ambulatorial da pressão arterial pode colaborar de maneira importante. 


\section{Hipertensão epi sódica}

A situação mais freqüente que retrata episódios isolados de hipertensão é a ocor rência do feocromocitoma. Ainda podemos observar picos hipertensivos em pacientes com síndrome de ansiedade ou de outras formas de desordens psíquicas.

\section{Síncope e hipotensão sintomática}

A ocorrência de episódios de hipotensão induzida por medicamentos ou mesmo de síncope pode perfeitamente ser detectada pela MAPA em 24 horas e dificilmente observada nas avaliações casuais.

\section{Avaliação da eficácia terapêutica anti-hipertensiva}

Muito se tem estudado ${ }^{17-19}$, nos últimos anos, a respeito da utilidade da MAPA na avaliação da efi cácia terapêutica das drogas anti-hi pertensi vas. Recentes publicações ${ }^{20-22}$ sobre variação pressórica nas 24 horas, bem como análise da eficácia das principais medicações utilizadas no tratamento da hipertensão, têm demonstrado a importância desses aspectos no prognóstico dos pacientes hipertensos. Recentemente, utilizamos a monitorização ambulatorial da pressão arterial em al gumas investigações ${ }^{23,24}$ com medicamentos anti-hipertensivos, objetivando a análise de sua eficácia terapêutica.

\section{CONSIDERAÇÕES FINAIS}

A MAPA é um procedimento que, efetivamente, se incorporou à nossa atividade clínica. A cada dia, maiores vão se tornando os benefícios que podem ser auferidos dessa fascinante metodologia. As crescentes publicações de novas informações, estudos prospectivos em andamento, a mai or difusão do método e, conseqüentemente, o melhor conhecimento a seu respei to farão com que tenhamos, num futuro muito próximo, uma visão muito mais adequada e judiciosa desse importante método.

O caminho do mel hor conhecimento da hipertensão arterial a respeito de sua fisiopatologia, diagnóstico, terapêutica e prognóstico é, seguramente, constituído pel o entendimento das variações dinâmicas da pressão arterial adequadamente estudadas pela monitorização ambulatorial da pressão arterial de 24 horas.

\section{REFERÊNCIAS BIBLIOGRÁFICAS}

1. National High Blood Pressure Education Program Working Group Report on Ambulatory Blood Pressure Monitoring. Arch Intern Med 1990; 150: 2.270-80.

2. Elliot HL. Arterial blood pressure monitoring. Annals of Second International Nifedipine GITS Simposium. Paris, 1994; $17-20$

3. Millar-Craig MW, Haves D, Whittington J. New system for recording ambulatory and blood pressure in man. Med Biol Eng Comput 1978;16: 727-31.
4. Nobre F, Mion J r D, Spritzer N, Oigman W. I Consenso Brasileiro para uso de monitorização ambulatorial da pressão arterial. Arq Bras Cardiol 1993; 60: 129-34.

5. SoK ol ow M, Werdegar D, Kain HK, Hinman AT. Relationship between levels of blood pressure casually and by portable recorders and severity of complications in essential hypertension. Circulation 1966; 34: 279-98.

6. Perloff $B$, Sokolow M, Cowan R. The prognostic value of ambulatory blood pressure monitoring in treated hypertensive patients. J Hypertens 1991; 9(suppl 1): S33-40.

7. Pickering TG, Devereux RB. Ambulatory monitoring of blood pressure as a predictor of cardiovascular risk. Am Heart J 1987; 114: 925-8.

8. White WB. Analysis of ambulatory blood pressure data in antihypertensive drug trials. J Hypertens 1991; 9(suppl 1): S27-32.

9. White WB, Morganroth J. Usefulness of ambulatory blood pressure monitoring in assessing antihypotensive therapy. Am J Cardiol 1989; 63: 94-8.

10. Nobre F, Nogueira J L. Tratamento da hipertensão arterial sistêmica com urapidil. Estudo na hipertensão leve e moderada com monitorização ambulatorial da pressão arterial. Arq Bras Cardiol 1993; 61: 63-8.

11. Garret NB, Kaplan N. Ambulatory blood pressure monitoring. A question for now and the future. J Clin Hipertens 1987; 3: 378-80.

12. Sheps SG, Pickering TG, White WB et al. ACC position statement: ambulatory blood pressure monitoring. J ACC 1994; 23, 6: 1.511-3.

13. Second International Consensus Meeting on 24 hour blood pressure ambulatory monitoring measurement: consensus and conclusions. J Hypertens 9(suppl 8), S2-26, 1991.

14. Second International Consensus Meeting on 24 hour blood pressure ambulatory monitoring measurement: consensus and convulsions. J Hypertens 1991; 9(suppl 8): S28-30.

15. Pickering TT, J ames J D. Some implications of the diferences between home, clinic and ambulatory blood pressure in normotensive and hypertensive patients. J Hypertens 1989; 7(suppl 3): S65- 72.

16. White WB. Average daily blood pressure, not office blood pressure, determines cardiac function in patients with hypertension. J AMA 1989; 261: 873-7.

17. Elliot J HL. Trough: peak ratio and twenty-four-hour blood pressure control. J Hypertens 1994; 12(suppl 5): S29-33.

18. Meredith PA. Trough: peak ratios for antihypertensive agents. The issues in perspective. Drugs 1994; 48(5): 661-6.

19. Parati G, Ravogli A, Frattola E. et al. Blood pressure variability: clinical implications and effects of antihypertensive treatment. J Hypertens 1994; 12(suppl 5): S35-40.

20. Parati G, Ravogli A, Gropelli E et al. Blood pressure variability: clinical implications and effects of antihypertensive treatment. J Hypertens 1994;12(suppl 5): 35-40.

21. Coca A. Circadian rhythm and blood pressure control: physiological and pathophysiological factors. J Hypertens 1994;12(suppl 5): S13-21.

22. Colin J S, Valente AJ . Hildebrandt R. Prevention of atherosclerosis and endorgan damage: a basis for antihypertensive interventional strategies.J Hypertens 1994; 12(suppl 5): S-3S11. (falta prenome últ. autor)

23. Nobre F, Oigman W. Avaliação da eficácia terapêutica de Diltiazen AP no tratamento da hipertensão leve e moderada, com monitorização ambulatorial da pressão arterial. Arq Bras Cardiol 1994; 63(4): 321-6.

24. Magliano MF, Amodeo C, Mion J r D et al. Estudo multicêntrico brasileiro de avaliação da eficácia clínica e tolerabilidade da isradipina SRO através de monitorização ambulatorial da pressão arterial no tratamento da hipertensão arterial leve e moderada. Arq Bras Cardiol 1993; 61(5): 311-8. 